\title{
Fatores associados ao nível de atividade física praticada por estudantes universitários
}

\author{
Factors associated to level of exercise practiced by university students \\ Luciana Gonzaga dos Santos Cardoso ${ }^{\circledR 1}$, Amanda Duarte dos Santos ${ }^{\circledR 1}$, \\ Rosimeire Angela Queiroz Soares ${ }^{\circledR 1}$, Livia Keismanas de Ávila ${ }^{\circledR 1}$
}

\begin{abstract}
Resumo
Objetivo: Identificar os fatores sociodemográficos relacionados com o nível de atividade física (AF) em estudantes universitários da área da saúde. Método: Estudo, descritivo e quantitativo, com estudantes de uma instituição de ensino superior privada, ocorrida no segundo semestre de 2016, referentes à identificação sociodemográfica e nível de AF praticada. Resultados: Participaram da pesquisa 87 estudantes, a maioria com idade inferior a 30 anos, sexo feminino, cor branca, solteiros/divorciados, com renda de até cinco salários mínimos. A autopercepção da saúde foi maior no curso de fonoaudiologia, e a restrição para AF foi maior entre estudantes de medicina. A média de dias/semana foi maior para a caminhada, seguida da atividade moderada e vigorosa em todos os cursos, exceção para o curso de medicina. Conclusão: Os fatores sociodemográficos relacionados ao nível de atividade física praticada pelos estudantes foram o curso e o número de pessoas que vivem da renda familiar.
\end{abstract}

Palavras chave: Exercício físico, Estudantes de ciências da saúde, Promoção da saúde

\section{Abstract \\ Objective: Identify the sociodemographic factors related with the level of exercises on health degree college students. Method: Study, descriptive and quantitative, with private university college students, during the second semester of 2016, referring to sociodemographic identification and level of exercise practiced. Results: 87 students participated in the research, mostly under 30 years old, female, white,}

1. Faculdade de Ciências Médicas da Santa Casa de São Paulo. Curso de Graduação em Enfermagem. São Paulo - SP - Brasil

Trabalho realizado: Faculdade de Ciências Médicas da Santa Casa de São Paulo. Curso de Graduação em Enfermagem. São Paulo-SP-Brasil

Endereço para correspondência: Luciana Gonzaga dos Santos Cardoso. Faculdade de Ciências Médicas da Santa Casa de São Paulo - Curso de Graduação em Enfermagem. Rua Dr. Cesário Motta Jr., 61 - Vila Buarque - 01221-020 - São Paulo - SP - Brasil. Fone: (11)3367-7798-E-mail: luciana.cardoso@fcmsantacasasp.edu.br single/ divorced, income up to five minimum wage (BR). Healthiness self-perception was higher in Speech pathology students, and restriction for exercise was higher on Medicine students. The average of days/week was higher for walking, followed by moderate activity and vigorous exercise on all courses, except by medicine. Conclusion: The sociodemographic factors related to exercise levels practiced by students was degree and number of people who live from the family income.

Keywords: Exercise; Students, health occupations; Health promotion

\section{Introdução}

O ingresso em um curso de graduação gera a necessidade de adaptação do estudante ao novo ambiente. As dificuldades e cobranças impostas pelo meio acadêmico e mudanças de rotina são frequentes. Carga horária de estudo extensa, mudanças no contexto social, de lazer, sobrecarga de trabalho e emocional estão presentes neste novo contexto e, portanto, o estudante poderá sofrer influência do próprio meio em que está inserido ${ }^{(1)}$. O nível de atividade física pode ser um destes fatores influenciados pelo ingresso na graduação ${ }^{(1-3)}$ Estudantes universitários da área da saúde compartilham o conhecimento dos benefícios que a atividade física gera à promoção da saúde e à prevenção de doenças crônicas não transmissíveis. A orientação aos usuários dos serviços de saúde quanto à necessidade da prática da atividade física faz parte das atividades desenvolvidas durante a graduação. Entretanto, a atividade física nem sempre é um hábito presente entre esses indivíduos ${ }^{(2-3)}$ Atividade física é definida por qualquer movimento produzido pelo corpo que gera gasto energético ${ }^{(4)}$. Pode ser laboral ou ocupacional, realizada no transporte, lazer ou atividades domésticas. Quando a atividade física ocorre de maneira estruturada, desde que planejada e repetitiva com a intenção da melhora da aptidão física, mesmo que por lazer, convencionou-se chamar de exercício físico $^{(4)}$. Estas atividades tem um efeito positivo sobre a qualidade de vida, condições físicas e psicológicas. Promovem melhora da autoestima, do autoconceito, 
da imagem corporal, da insônia, das funções cognitivas e da socialização, incremento da massa e da força muscular, contribui para a melhora da auto-eficácia, da tensão muscular, favorece a perda de peso, diminui o estresse, a ansiedade e o consumo de medicamentos ${ }^{(5-6)}$. A atividade física gera estresse fisiológico por aumentar a demanda energética em relação ao repouso. Ao longo do tempo, o corpo se adapta às alterações estruturais e funcionais decorrentes da atividade física, aumenta a capacidade de responder ao estresse e os níveis de HDL, retarda o processo aterosclerótico, reduz o vaso espasmo, aumenta a estabilidade elétrica do miocárdio, aumenta a fibrinólise e reduzir os níveis de triglicerídeos ${ }^{(6)}$. A prática de atividade física é capaz de prevenir doenças crônicas e reduzir o risco de mortalidade e morbidade, independente de outras mudanças no estilo de vida. Sabe-se que aproximadamente 30 minutos de atividade física, pelo menos três vezes por semana pode ser útil para reduzir o risco de eventos cardiovasculares ${ }^{(8)}$. Por outro lado, atividades rotineiras como caminhar, subir escadas e andar de bicicleta estão sendo substituídas pelo aumento de atividades sedentárias como uso de carro para transporte, uso de escadas rolantes, televisão, videogames e computadores ${ }^{(9)}$. A inatividade física está associada à maior prevalência de doenças cardiovasculares, câncer de colón, depressão, dislipidemia, diabetes, osteoporose e obesidade. Estas doenças aumentam significativamente a procura por atendimento médico e maior dependência de terceiros quando em idades avançadas ${ }^{(9)}$. Atualmente as doenças cardiovasculares são as principais causas de óbitos no Brasil e no mun$\mathrm{do}^{(7-8,10)}$ Apesar dos alunos demonstrarem conhecimento dos possíveis benefícios físicos e sociais da atividade física, como por exemplo, o aumento da capacidade de concentração, rendimento acadêmico e maior perseverança na realização de tarefas, alguns estudos com universitários demonstram a diminuição na prática da atividade física após o ingresso à universidade ${ }^{(1-3)}$. Entre as justificativas, os alunos relatam a sobrecarga horária de aulas e maior dificuldade em abdicar do tempo livre, que frequentemente é utilizado para festas e confraternizações como meio de uma distração psicológica. Em contrapartida, estudantes que praticam atividade física moderada frequentemente relatam relaxamento psicológico, diminuição dos desconfortos em relação a vida académica e efeitos benéficos para a saúde física ${ }^{(1,3)}$. O enfermeiro desempenha um importante papel no cuidado em saúde, tanto na promoção como na manutenção da saúde, contribuindo para o aprendizado sobre prevenção de doenças, métodos ambientais saudáveis e sobre a utilização dos serviços de saúde ${ }^{(11)}$.

Espera-se que este estudo proporcione conhecimentos úteis para o estabelecimento de estratégias que favoreçam o aumento da prática da atividade física entre os estudantes.

\section{Objetivo}

Identificar os fatores sóciodemográficos que se relacionam com o nível de atividade física praticada por estudantes universitários da área da saúde.

\section{Material e Método}

Pesquisa prospectiva, descritiva e de análise quantitativa dos dados, realizada com discentes dos Cursos de Graduação em Enfermagem, Fonoaudiologia, Medicina, Tecnologia em Radiologia e Tecnologia em Sistemas Biomédicos de uma instituição de ensino superior, privada, localizada na região central da cidade de São Paulo. Os dados foram coletados no segundo semestre de 2016, por meio de formulários eletrônicos enviados aos participantes por e-mail, utilizando-se a ferramenta Google Docs ${ }^{\circledR}$. O projeto foi aprovado pelo Comitê de Ética em Pesquisa, conforme parecer CAAE: 56071316.6.0000.5479 e os participantes concordaram em participar do estudo mediante o aceite no Termos de Consentimento Livre e Esclarecido (TCLE). Foram coletados: idade, sexo, cor, local de residência, estado civil, filhos, trabalho, renda familiar, número de pessoas que vivem com a renda, curso de graduação, auto percepção da saúde, restrição para realização de atividade física, número de dias na semana que pratica atividade física e intensidade da atividade, classificada em caminhada, atividade moderada ou atividade vigorosa. As informações foram inseridas em um banco de dados do programa Microsoft Excel ${ }^{\circledR}$ e analisadas pela estatística descritiva e analítica no programa EpiInfo $^{\circledR}$, versão 7.2.2.16. As variáveis qualitativas, foram apresentadas em frequência absoluta $(\mathrm{N})$ e relativa (\%), com auxílio de tabelas, e para as variáveis quantitativas foi calculada a média e o desvio padrão. As associações com os dados sociodemográficos foram obtidas com testes analíticos e atribuída significância estatística para valores de $p<0,05$.

\section{Resultados}

Participaram da pesquisa 87 de cerca de 1200 estudantes matriculados no período de coleta de dados, a amostra representou $7,2 \%$ de todos os estudantes dos cinco cursos de graduação. Prevaleceram os estudantes com idade menor que 30 anos $(81,4 \%)$, do sexo feminino $(71,3 \%)$, cor branca $(71,3 \%)$, solteiros/divorciados $(91,9 \%)$, com renda familiar de até cinco salários mínimos (48,3\%). A autopercepção da saúde foi maior no curso de fonoaudiologia, seguida dos cursos de medicina, enfermagem e tecnológicos. A restrição para realização de AF foi maior entre os estudantes de medicina. Entre os que praticam AF, a maioria dos estudantes de todos os cursos relatam 
praticar atividade física de quatro a sete dias por semana. A Tabela 1 apresenta informações sociodemográficas, autopercepção de saúde, restrição para AF e prática de AF.

$\mathrm{Na}$ amostra geral, a média da prática de AF foi de 5,09 dias por semana com desvio padrão de 1,7. A Ta- bela 2 mostra a associação entre a média da prática de AF em dias por semana segundo o curso que estudante frequenta, as variáveis sociodemográficas, a restrição para realização de atividade física e a autopercepção de saúde, e mostra que houve diferença estatisticamente significante para os cursos e para o número de

\section{Tabela 1}

Frequência absoluta (n) e relativa (\%) das variáveis sociodemográficas, autopercepção de saúde, restrições para realização de atividade física e prática de atividade física em dias por semana, segundo o curso. n=87 (São Paulo, 2016)

\begin{tabular}{|c|c|c|c|c|}
\hline & $\begin{array}{c}\text { Enfermagem } \\
n(\%)\end{array}$ & $\begin{array}{c}\text { Fonoaudiologia } \\
n(\%)\end{array}$ & $\begin{array}{c}\text { Medicina } \\
n(\%)\end{array}$ & $\begin{array}{c}\text { Tecnológicos } \\
n(\%)\end{array}$ \\
\hline \multicolumn{5}{|l|}{ Faixa etária (anos) } \\
\hline 18 a 29 & $20(74,1)$ & $11(84,6)$ & $33(84,6)$ & $6(85,7)$ \\
\hline$\geq 30$ & $7(25,9)$ & $2(15,4)$ & $6(15,4)$ & $1(14,3)$ \\
\hline \multicolumn{5}{|l|}{ Sexo } \\
\hline Feminino & $19(70,4)$ & $8(61,5)$ & $30(75,0)$ & $5(71,4)$ \\
\hline Masculino & $8(29,6)$ & $5(38,5)$ & $10(25,0)$ & $2(28,6)$ \\
\hline \multicolumn{5}{|l|}{ Cor } \\
\hline Branca & $20(74,1)$ & $8(61,5)$ & $30(75,0)$ & $4(57,1)$ \\
\hline Parda/Preta & $6(22,2)$ & $5(38,5)$ & $8(20,0)$ & $3(42,9)$ \\
\hline Amarela & $1(3,7)$ & - & $2(5,0)$ & - \\
\hline \multicolumn{5}{|l|}{ Residência } \\
\hline São Paulo & $23(85,2)$ & $10(76,9)$ & $35(87,5)$ & $6(85,7)$ \\
\hline Outros & $4(14,8)$ & $3(23,1)$ & $5(12,5)$ & $1(14,3)$ \\
\hline \multicolumn{5}{|l|}{ Estado civil } \\
\hline Solteiro/Divorciado & $23(85,2)$ & $13(100,0)$ & $39(97,5)$ & $5(71,4)$ \\
\hline Casado/União estável & $4(14,8)$ & - & $1(2,5)$ & $2(28,6)$ \\
\hline \multicolumn{5}{|l|}{ Filhos } \\
\hline Não & $22(81,5)$ & $12(93,3)$ & $36(90.0)$ & $6(85,7)$ \\
\hline Sim & $5(18,5)$ & $1(7,8)$ & $4(10,0)$ & $1(14,3)$ \\
\hline \multicolumn{5}{|l|}{ Trabalha } \\
\hline Não & $18(66,7)$ & $8(61,5)$ & $30(75,0)$ & $5(71,4)$ \\
\hline Sim & $9(33,3)$ & $5(38,5)$ & $10(25,0)$ & $2(28,6)$ \\
\hline \multicolumn{5}{|l|}{ Renda familiar (SM) } \\
\hline$\leq 5$ & $12(44,5)$ & $6(46,2)$ & $19(47,5)$ & $5(71,4)$ \\
\hline$>5<10$ & $6(22,2)$ & $1(7,6)$ & $9(22,5)$ & - \\
\hline$\geq 10$ & $9(33,3)$ & $6(46,2)$ & $12(30,0)$ & $2(28,6)$ \\
\hline \multicolumn{5}{|l|}{ Pessoas que vivem da renda } \\
\hline$\geq 4$ & $14(51,8)$ & $9(69,2)$ & $23(57,5)$ & $4(57,1)$ \\
\hline 1 a 3 & $13(48,2)$ & $4(30,8)$ & $17(42,5)$ & $3(42,9)$ \\
\hline \multicolumn{5}{|l|}{ Autopercepçãoda saúde } \\
\hline Boa & $18(66,7)$ & $4(30,8)$ & $13(32,5)$ & $5(71,4)$ \\
\hline Muito boa & $7(25,9)$ & $3(23,1)$ & $21(52,5)$ & - \\
\hline Excelente & $2(7,4)$ & $6(46,1)$ & $6(15,0)$ & $2(28,6)$ \\
\hline \multicolumn{5}{|l|}{ Restrição para AF } \\
\hline Não & $26(96,3)$ & $12(93,3)$ & $35(87,5)$ & $7(100,0)$ \\
\hline Sim & $1(3,7)$ & $1(7,7)$ & $5(12,5)$ & - \\
\hline \multicolumn{5}{|c|}{ Prática de AF (dias/semana) } \\
\hline Zero & $2(7,4)$ & - & $1(2,5)$ & $2(28,6)$ \\
\hline 1 a 3 & - & $1(7,7)$ & $7(17,5)$ & - \\
\hline 4 a 7 & $25(92,6)$ & $12(92,3)$ & $32(80,0)$ & $5(71,4)$ \\
\hline
\end{tabular}


Tabela 2

Associação da média de dias por semana da prática de AF com curso, variáveis sociodemográficas, a restrição para realização de atividade física e a autopercepção de saúde. $n=87$ (São Paulo, 2016)

\begin{tabular}{|c|c|c|}
\hline AF em dias/semana & Média $(D P)$ & $\begin{array}{l}p \text {-valor } \\
(\leq 0,05)\end{array}$ \\
\hline \multicolumn{3}{|l|}{ Curso } \\
\hline Enfermagem & $5,8(1,8)$ & \\
\hline Fonoaudiologia & $4,7(0,9)$ & \\
\hline Medicina & $4,8(1,5)$ & \\
\hline Tecnológicos & $4,2(3,0)$ & 0,002 \\
\hline \multicolumn{3}{|l|}{ Faixa etária (anos) } \\
\hline 18 a 29 & $4,9(1,8)$ & \\
\hline$\geq 30$ & $5,5(1,2)$ & 0,299 \\
\hline \multicolumn{3}{|l|}{ Sexo } \\
\hline Feminino & $5,2(1,7)$ & \\
\hline Masculino & $4,8(1,8)$ & 0,331 \\
\hline \multicolumn{3}{|l|}{ Cor } \\
\hline Branca & $5,1(1,6)$ & \\
\hline Parda/Preta & $5,2(1,7)$ & \\
\hline Amarela & $2,6(3,0)$ & 0,051 \\
\hline \multicolumn{3}{|l|}{ Residência } \\
\hline São Paulo & $5,0(1,8)$ & \\
\hline Outros & $5,1(1,2)$ & 0,892 \\
\hline \multicolumn{3}{|l|}{ Estado civil } \\
\hline Solteiro/Divorciado & $5,0(1,6)$ & \\
\hline Casado/União estável & $5,1(2,7)$ & 0,937 \\
\hline \multicolumn{3}{|l|}{ Filhos } \\
\hline Não & $5,1(1,7)$ & \\
\hline Sim & $5,0(2,0)$ & 0,854 \\
\hline \multicolumn{3}{|l|}{ Trabalha } \\
\hline Não & $5,0(1,75)$ & \\
\hline Sim & $5,0(1,83)$ & 0,959 \\
\hline \multicolumn{3}{|l|}{ Renda familiar (SM) } \\
\hline$\leq 5$ & $5,2(1,4)$ & \\
\hline$>5<10$ & $4,8(2,1)$ & \\
\hline$\geq 10$ & $5,0(1,45)$ & 0,793 \\
\hline \multicolumn{3}{|c|}{ Pessoas que vivem da renda } \\
\hline 1 a 3 & $5,6(1,1)$ & \\
\hline$\geq 4$ & $4,7(2,0)$ & 0,039 \\
\hline \multicolumn{3}{|l|}{ Restrição para AF } \\
\hline Não & $5,1(1,6)$ & \\
\hline Sim & $4,2(2,5)$ & 0,210 \\
\hline \multicolumn{3}{|l|}{ Autopercepção da saúde } \\
\hline Boa & $5,0(2,2)$ & \\
\hline Muito boa & $5,2(1,6)$ & \\
\hline Excelente & $5,0(1,6)$ & 0,716 \\
\hline
\end{tabular}

pessoas que vivem da renda familiar.

Os estudantes também responderam sobre a intensidade da atividade física que realizavam (caminhada, moderada ou vigorosa). A média de dias / semana que realizam $\mathrm{AF}$ foi maior para a caminhada, seguida da atividade moderada e vigorosa em todos os cursos, exceção apenas para o curso de medicina, onde a média de AF moderada e vigorosa foram muito semelhantes. A Tabela 3 apresenta a associação entre a intensidade da atividade física e os cursos que os estudantes frequentam, e mostra que houve diferença estatisticamente significante entre os cursos na prática da caminhada.

\section{Discussão}

Observa-se uma predominância do sexo feminino, o que já era de se esperar já que atualmente no Brasil indicadores mostram que no ensino universitário a presença das mulheres ainda é predominante. Além disso, enfermagem e fonoaudiologia são profissões exercidas, na maioria, por mulheres ${ }^{(12)}$. Neste sentido, a prevalência de mulheres pode estar associada aos resultados identificados com relação ao sexo. Por outro lado, as exigências quanto ao corpo feminino também fazem com que as mulheres mais jovens optem por realização de atividade física, com objetivo de modelar o corpo. Observou-se que os indivíduos de melhor renda e branco foram os que mais realizavam $\mathrm{AF}$, compatíveis com dados da literatura, em que fatores cor e renda ainda são determinantes para o ingresso de jovens no ensino superior, conforme verificado também por outros pesquisadores ${ }^{(13)}$. Apesar do número de estudantes autodeclarados brancos estar diminuindo desde 2004, eles continuam sendo maioria nas universidades ${ }^{(13)}$. Quanto à renda familiar, atualmente existem programas que permitem um maior acesso de classes sociais menos favorecidas à universidade. Quando observamos os cursos da área da saúde, com carga horaria elevada e em período diurno, percebemos que muitas vezes os discentes não possuem tempo suficiente para os estudos e disposição para uma jornada de trabalho ${ }^{(14)}$. Esta intensa carga horária inviabiliza disponibilização de tempo para realização de AF. Quanto à faixa etária houve predomínio de estudantes com idade menor que 30 anos. Tais resultados corroboram os achados da literatura possivelmente decorrente do impacto da política de expansão do ensino superior impulsionada pelo Plano Nacional de Educação. No que se refere ao estado civil houve predominância de discentes que se declararam solteiros, dado compatível com a idade média da amostra, além disto, sabe-se que jovens solteiros tem uma maior disponibilidade de tempo e dedicação à intensa carga horária dos cursos superiores $^{(15)}$. A autopercepção da saúde foi maior no curso de fonoaudiologia, seguida dos cursos de medicina, enfermagem e tecnológicos. Segundo alguns pesquisadores a autopercepção negativa do seu próprio estado de saúde pode relacionar-se à di- 


\section{Tabela 3}

Associação entre intensidade da prática de atividade física, apresentada em média e desvio padrão de dias da semana, e cursos da área da saúde. $n=87$ (São Paulo, 2016)

\begin{tabular}{lccccc} 
& $\begin{array}{c}\text { Enfermagem } \\
\text { média(DP) }\end{array}$ & $\begin{array}{c}\text { Fonoaudiologia } \\
\text { média(DP) }\end{array}$ & $\begin{array}{c}\text { Medicina } \\
\text { média(DP) }\end{array}$ & $\begin{array}{c}\text { Tecnológicos } \\
\text { média(DP) }\end{array}$ & $\begin{array}{c}p \text {-valor } \\
(\leq 0,05)\end{array}$ \\
Caminhada (dias/semana) & $5,7(1,8)$ & $4,6(0,8)$ & $4,1(2,3)$ & $4,2(3,0)$ & $\mathbf{0 , 0 0 0}$ \\
AF Moderada (dias/semana) & $2,7(2,3)$ & $2,2(2,0)$ & $2,0(1,6)$ & $2,7(2,9)$ & 0,561 \\
AF Vigorosa (dias/semana) & $1,6(1,9)$ & $0,9(1,2)$ & $2,1(1,7)$ & $0,7(0,9)$ & 0,057 \\
\hline
\end{tabular}

minuição da pratica de atividade física, reforçando a ideia de que a atividade física impacta positivamente a percepção de saúde ${ }^{(16)}$. Neste sentido, a restrição para realização de AF foi maior entre os estudantes de medicina. Com relação ao tempo de AF, embora com diferença estatisticamente significante entre eles $(p=0,002)$; a maioria dos estudantes, independente do curso, relatou praticar atividade física de quatro a sete dias por semana. Este estudo também mostrou diferença estatisticamente significante na prática de atividade física em relação ao número de pessoas que vivem da renda familiar, ou seja, observou-se que a prática da $\mathrm{AF}$ está diretamente ligada ao menor número de pessoas que vivem da renda $(p=0,039)$. Se por um lado, a educação e o nível socioeconômico são entendidos como fatores sociais diretamente ligados ao nível de atividade física, assim, quanto maior o nível de instrução e nível socioeconômico maior deveria ser o entendimento dos benefícios da atividade física e qualidade de vida no geral ${ }^{(16)}$. Por outro lado, um outro estudo mostrou que maior renda familiar pode diminuir as oportunidades de realização de atividade física, uma vez que a melhor condição sócio econômica facilita o acesso a hábitos que diminuem a necessidade de realização de atividade física, tais como o uso de veículos para se locomover, ao invés da caminhada; utilização de controle remoto, aumentando o sedentarismo, acesso à Internet, bem como a terceirização de serviços domésticas; corroborando para os baixos níveis de atividade física entre os universitários ${ }^{(17)}$. Com exceção da medicina, onde a média de AF moderada e vigorosa foram muito semelhantes, o tipo de atividade física mais praticada entre os estudantes dos demais cursos neste estudo foi a caminhada, seguida da atividade moderada e vigorosa. A necessidade de dedicar-se várias horas curriculares e extracurriculares nos cursos superiores pode estar relacionada ao maior estresse e menor disponibilidade de tempo para realização de atividades físicas, uma vez que esta prática demanda uma maior disponibilidade de tempo ${ }^{(14)}$. Sabe-se que os estudantes estão continuamente sujeitos a trabalhos, prazos de entrega, longas horas de estudo, situações extenuantes e estressantes que contribuem para sua exaustão física e mental ${ }^{(15)}$. Sabe-se que a adoção de práticas esportivas nos tempos livres, melhora a qualidade de vida e bem-estar dos estudantes universitários, diminuindo queixas de problemas relacionados à saúde ${ }^{(16)}$. Sendo assim, os resultados do presente estudo são relevantes, consistentes e representativos. A Universidade deve servir para a disseminação de saberes, cultura e valores, dentre os quais as atividades físicas não podem estar excluídas. No meio acadêmico as dificuldades e cobranças impostas para garantir um bom desempenho, situações de exaustão física, mental e mudanças de rotina são frequentes, tudo isto podendo influenciar na pratica de atividade física. Conhecer os hábitos relacionados à pratica de atividade física entre estudantes universitários é importante para que possam ser planejadas e implementadas medidas que corroborem para a adoção de hábitos saudáveis de vida. E papel da Universidade auxiliar o aluno a se desenvolver permanentemente, através da disseminação de saberes, valores e culturas, que contribuam para o crescimento profissional, pessoal, físico e mental dos acadêmicos. Neste contexto, a prática de atividade física, diante dos inúmeros e irrefutáveis benefícios que traz aos indivíduos, frente às necessidades emergentes dos próprios acadêmicos, deve não somente ser recomendada e reconhecida, mas estimulada.

São limitações deste estudo o número de participantes em relação ao número de alunos matriculados no momento da coleta de dados e a falta do dado tempo de "atividade física praticada". Novas pesquisas devem ser conduzidas para atender a estas limitações.

\section{Conclusão}

A prática da atividade física traz inúmeros benefícios à saúde e por isso deve ser incentivada no ambiente universitário. Os fatores sociodemográficos que se relacionaram com o nível de atividade física praticada por estudantes universitários da área da saúde foram o curso e o número de pessoas que vivem da renda familiar. Houve diferença significante para média de dias da semana de prática da caminhada entre os cursos. Conhecer estes fatores possibilitam subsidiar pesquisas futuras para promover e estimular esta prática entre este grupo específico. 


\section{Referências}

1. Souza LK, Lourenço E, Santos MRG. Adaptação à universidade em estudantes ingressantes na graduação em psicologia. Psicol Educ. 2016; 42(1):35-48.

2. Jesus CF, Oliveira RAR, Badaró AC, Ferreira EF. Nível de atividade física de estudantes da área da saúde de uma instituição superior particular de Ubá-MG. Revista Brasileira de Prescrição e Fisiologia do Exercício. [Internet]. 2017 [citado 2018 Mar 21];11(68):565-73. Disponível em: https://www. semanticscholar.org/paper/N\%C3\%ADvel-de-atividadef\%C3\%ADsica-de-estudantes-da- $\%$ C $3 \%$ A 1 rea-da-JesusOliveira/a82dc09d0cb811d194fb221a7d758f8c99a8c913

3. Nunes CCG. Atividade física dos estudantes de enfermagem: determinantes sociodemográficos e psicológicos. Dissertação (Mestrado). Viseu: Instituto Politécnico de Viseu; 2015.

4. Matsudo S, Araújo T, Matsudo V, Andrade D, Andrade E, Oliveira LC, Braggion G. Questionário Internacional de Atividade Física (IPAQ): estudo de validade e reprodutibilidade no Brasil. Rev Bras Ativ Dis Saúde. 2001; 6(2):5-18.

5. Ferreira JS, Diettrich SHC, Pedro DA. Influência da prática de atividade física sobre a qualidade de vida de usuários do SUS. Saúde Debate. [Internet]. 2015 [citado 2018 Mar 20]; 39(106):792-801.5. Disponível em: https://www.scielo.br/ scielo.php?script=sci_arttext\&pid=S0103-11042015000300792.

6. Coelho FGM, Virtuoso Júnior JS. Atividade física e saúde mental: o que precisamos entender sobre promoção, prevenção e tratamento? Rev Enferm Atenção Saúde. [Internet]. 2015 [citado 2018 Mar 20]; 4(2):1-4. Disponível em: http://seer.uftm.edu.br/ revistaeletronica/index.php/enfer/article/view/1547

7. Marques TS, Heubel AD, Gimenes C, Arca EA, Pessoa-Santos BV, Martinelli B, et al. Lipid profile, functional capacity and ankle-brachial index in active older adults. Rev Bras Med Esporte. [Internet]. 2019 [citado 2018 Mar 20]; 25(4):316-21. Disponível em: https:/ /www.scielo.br/scielo.php?script=sci_ar ttext\&pid=S1517-86922019000400316

8. Pereira DS, Souza SJP, Marques FR, Weigert SP. A atividade física na prevenção das doenças crônicas não transmissíveis. [Internet]. 2017 [citado 2019 Mar 20]; Rev Gest Saúde. 17 (Supl 1):S1-9. Disponível em: http://www.herrero.com.br/files / revista/fileaf5bd92eab28b9ad78efcd42cfde9745.pdf

9. Bernardo AFB, Rossi RC, Souza NM, Pastre CM, Vanderlei LCM. Associação entre atividade física e fatores de risco cardiovasculares em indivíduos de um programa de reabilitação cardíaca. Rev Bras Med Esporte. [Internet]. 2013 [citado 2019 Mar 21]; 19(4):231-5. Disponível em: https:/ / www.scielo.br/ scielo.php?script=sci_arttext\&pid=S1517-86922013000400001

10. Booth FW, Roberts CK, Thyfault JP, Ruegsegger GN, Toedebusch RG. Role of inactivity in chronic diseases: evolutionary insight and pathophysiological mechanisms. Physiol Rev. 2017; 97(4):1351-402.

11. Bastable SB. O enfermeiro como educador. Princípios de ensinoaprendizagem para a prática de enfermagem. $3^{\mathrm{a}}$. ed. Porto Alegre: Artmed; 2008. 688p.

12. Barreto A. A mulher no ensino superior distribuição e representatividade. Cadernos do GEA. [Internet]. 2014 [citado 2019 Jan 10]; (6):1-46. Disponível em: https://flacso.org.br/ files/2016/04/caderno_gea_n6_digitalfinal.pdf

13. Correa AK, Prebill GM , Ruiz JC, Souza MCB, Santos RA. O perfil do aluno ingressante em um Curso de Bacharelado e Licenciatura em Enfermagem de uma Instituição de Ensino Superior Pública. Educ Rev. [Internet]. 2018 [citado 2019 Mar 21]; 34:e185913|. Disponível em: https://www.scielo. $\mathrm{br} /$ scielo.php?pid=S0102-46982018000100146\&script=sci abstract\&tlng=pt.

14. Marcondelli P, Costa T H, Schmitz B. Nível de atividade física e hábitos alimentares de universitários do $3^{\circ}$ ao $5^{\circ}$ semestres da área da saúde. Rev Nutr. 2008; 21(1):39-47.

15. Fontes A D, Vianna R PT. Prevalência e fatores associados ao baixo nível de atividade física entre estudantes universitários de uma universidade pública da região Nordeste - Brasil. Rev Bras Epidemiol. [Internet]. 2009 [citado 2020 Jan 10]; 12(1):20-9. Disponível em: https: / / www.scielo.br/scielo.php?pid=S1415790X2009000100003\&script=sci_abstract\&tlng=pt

16. Costa PHV, Silva FS, Machado CJ. Nível de atividade física e qualidade de vida dos estudantes de fisioterapia de uma instituição privada de ensino superior. Revista Interdisciplinar Ciências Médicas. 2018; 1(2):46-53.

17. Baretta E, Baretta M, Peres KG. Nível de atividade física e fatores associados em adultos no Município de Joaçaba, Santa Catarina, Brasil. Cad Saúde Pública. 2007; 23(7):1595-602.

Trabalho recebido: $27 / 04 / 2021$

Trabalho aprovado: $15 / 06 / 2021$

Trabalho publicado: 16/06/2021 